\title{
Avaliação dos egressos do curso de especialização em Saúde da Família no Espírito Santo, Brasil
}

\author{
Evaluation of egressing students of the specialization course \\ on Family Care H ealth in Espírito Santo State, Brazil
}

\author{
Ethel Leonor Noia M aciel ${ }^{1}$ \\ Pryscilla Formiga Figueiredo ${ }^{2}$ \\ Thiago N ascimento do Prado ${ }^{3}$ \\ H eleticia Scabelo Galavote ${ }^{4}$ \\ M aria Cristina Ramos ${ }^{5}$ \\ Maristela Dalbello de Araújo ${ }^{6}$ \\ Rita de Cássia Duarte Lima ${ }^{6}$
}

${ }^{1} \mathrm{Núcleos}$ deEstudos em Saúde Coletiva, UniversidadeFederal do Espírito Santo. Av. M arechal Campos 1.468, M aruípe. 29040-091 Vitória ES. emaciel@ndi.ufes.br

${ }^{2}$ Centro Biomédico, UniversidadeFederal do Espírito Santo.

${ }^{3}$ Laboratório de Epidemiologia, Núcleo de Doenças Infecciosas, UniversidadeFederal do

Espírito Santo.

${ }^{4}$ Prefeitura M unicipal de

Angra dos Reis.

${ }^{5}$ Departamento de

Enfermagem, Universidade

Federal do Espírito Santo.

${ }^{6}$ Programa de Pós-

graduação em Saúde

Coletiva, U niversidade

Federal do Espírito Santo.
Abstract The purpose of this study was to evaluate the contribution of the specialization course on Family Health Care (PG-PSF), in Espírito Santo State, for the reorientation of the practices in health. It is a cross-sectional study with a population of 47 egressing students of the PG-PSF coursein 2007. It wasused a semi-structured questionnaire for data collection data. The analysis of the generated contributions showed some reports regarding the changes in the work actions after the acquired knowledge during the course. The majority of thestudents declared that theacquired knowledge have applicability in their daily work. They also declared that can plan their actions based in the epidemic profile of the area where worked. The relevance of this course was verified for the egressing students, with the perspective of a constant adaptation of the contents and improvement of the didactic-pedagogic process, through a system of continuous education or permanent education, as well as the attendance processes and supervision in service.

Key words Egressing students, Continued education, $\mathrm{H}$ ealth service
Resumo Este estudo tem por objetivo descrever 0 perfil dos egressos e avaliar a contribuição do curso de pós-graduação lato sensu em Saúde da Família (PG-PSF), no Estado do Espírito Santo, para a reorientação das práticas em saúde. É um estudo do tipo descritivo de corte transversal, sendo a amostra composta por quarenta e sete egressos do curso PG-PSF em 2007. Para a coleta de dados, aplicou-se um questionário semiestruturado, contendo questões abertas e fechadas. A análise das contribuições geradas revelou mudanças nas ações de trabalho após os conhecimentos adquiridos durante o curso. A maioria afirmou que tais conhe cimentos têm aplicabilidade no cotidiano do trabalho e grande parte dos entrevistados conseguiu, apóso curso, planejar suasações com baseno perfil epidemiológico da área na qual atua. Constatousea relevância do curso para a capacitação profissional dos alunos, com a constante adequação dos conteúdos do processo didático-pedagógico, através de uma consistente política de educação permanente que tenha impactos e incorporação nos processos detrabalho, além da necessidade de programas de educação continuada que dêem conta das necessi dades específicas de cada prática profissional e da institucionalização dos processos de avaliação e autoavaliação como parte inseparável da organização do trabalho.

Palavras-chave Egressos, Educação continuada, Serviços de saúde 
Introdução

O processo de avaliar pressupõe um julgamento de valor que auxilia a tomada de decisões ${ }^{1}$. Se gundo Akerman e Luiz², a avaliação é parte importante na implementação de projetos e programas. Uma boa avaliação fornece informação sobre o que se está fazendo, se os objetivos estão sendo alcançados e se apontam novas estratégias. Constitui-se num processo contínuo, encorajando a reflexão sobre resultados e dando base para estratégias futuras. Acrescentando, $\mathrm{H}_{\text {artz }}{ }^{1}$ afirma que, se a avaliação for usada em um processo permanente, tendea aumentar a pertinência, a eficiência e a eficácia das atividades de saúde. A ausência de controles e metodologias de avaliação, em geral, leva a um gasto social ineficientee, consequentemente, ao desperdício dos re cursos disponíveis ${ }^{3}$.

Neste sentido, as metodologias de avaliação devem ser parte da agenda e das estratégias permanentes das Secretarias de Saúde, de forma a dar visibilidade e identificar as dificuldades, os nós críticos, as concepções, os processos de trabalho e os diferentes conceitos e práticas, como também as experiências positivas que vêm se consolidando em cenários como os municípios. Assim, é positiva a política do M inistério da Saúde de tornar essa ação como parte cotidiana dos processos de trabalho nas instituições. Além disso, deve-se apontar a relevância do processo de avaliação das instituições educacionais, dado o compromisso destas com a solução dos diversos prismas sociais, pela sua função de produtora e reprodutora de conhecimentos necessários para satisfazer as necessidades do meio em que estão inseridas e pelo seu financiamento público ${ }^{4}$.

O avanço da organização dos serviços de saúde no Brasil, na perspectiva da construção do Sistema Ú nico de Saúde (SUS) , traz novos desafios para a capacitação dos profissionais de saúde pública que atuam nos diversos serviços de saúde. A construção do SUS, mais precisamente a criação do Programa Saúde da Família (PSF), tem apontado a necessidade do investimento nos trabal hadores da saú de em várias dimensões, em particular no que diz respeito à educação permanente, quevisa, além da mel horia dos serviços de saúde, a al cançar níveis de qualidade de vida almejados pela população. N esse sentido, o M inistério da Saúdetem implementado várias iniciativas através do financiamento de cursos de pósgraduação dos trabalhadores de saúde, uma vez que estes profissionais são os atores principais na construção do sistema voltado para a presta- ção de serviço à população ${ }^{5}$. Dos cursos ofertados, destacamos a Especialização em Saúde da Família e Gestão do Sistema e dos Serviços de Saúde no SUS.

No Estado do Espírito Santo, a partir da necessi dade dese promoverem melhores condições técnicas às Equipes de Saúde da Família, foram construídas experiências distintas, primeiro através do Pólo de Capacitação, Formação e Educação Permanenteem Saúde da Família e, segundo, através do Pólo deEducação Permanenteem Saúde(PEPSUS).

A primeira experiência produziu um curso elaborado por profissionais das instituições que integravam o pólo: Secretaria de Estado da Saúde(SESA), UniversidadeFederal do Espírito Santo (UFES) eEscola de M edicina da Santa Casa de Misericórdia de Vitória (EM ESCAM), além de monitores dos cursos básicos de Saúde da Família, contando-se com a experiência dos pólos de vários estados, especialmente Paraná (Departamento de Saúde Comunitária da Universidade Federal do Paraná), sob a responsabilidade da UFES.

Além disso, foi realizado outro curso que, além dos profissionais que integravam o pólo do Estado do Espírito Santo, contou com a parceria e a responsabilidade da Escola Nacional de Saúde Pública-Fundação Oswaldo Cruz (ENSP-FIOCRUZ). Essas instituições estabeleceram um consórcio, visando a operacionalizar a formação em saúde da família no Estado do Espírito Santo, potencializando suas próprias experiências.

$\mathrm{N}$ a segunda experiência, foi construída uma proposta pedagógica única com as instituições de ensino vinculadas ao PEPSU S à SESA, com quatro turmas, cada uma sob a responsabilidadedeuma instituição deensino: UFES, Universidade de Vila Velha (UVV); EMESCAM e Faculdade Espírito-Santense (FAESA).

Considerando a relevância do processo de avaliação, o PEPSU S, em seu plano de ação, estabeleceu como uma de suas atividades uma pesquisa para avaliar a contribuição do curso de pós-graduação lato sensu em Saúde da Família no Estado do Espírito Santo para esses profissionais e as mudanças de suas práticas em saúde.

\section{Metodologia}

Realizou-se um estudo do tipo descritivo de corte transversal. No primeiro momento, foram analisados os registros de todos os egressos de cursos de especialização realizados pelo M inisté 
rio da Saúde no Estado do Espírito Santo a partir de 2004. No entanto, devido à grande mobilidade dos egressos e à não atualização dos cadastros, foi possível localizar, em número considerável, apenas os egressos de cursos realizados entre2006 e2007.

Para a realização deste estudo, foram convocados todos os egressos do curso de pós-graduação lato sensu em Saúde da Família em 2006 e 2007 no Estado do Espírito Santo, ofertado por quatro instituições de ensino superior, sendo três privadas e uma pública.

Para a coleta de dados, aplicou-se um questionário semiestruturado, conten do questões abertas e fechadas. As variáveis a serem avaliadas foram divididas em dados de identificação, formação, produtos efetuados, trabal ho atual e impacto do curso.

Dos cem alunos finalistas, cujos endereços foram localizados, 47 (47\%) devolveram o questionário preenchido e fizeram, então, parte da amostra do estudo.

Antes de receberem os questionários, os participantes foram informados sobre os objetivos da pesquisa, com a disponibilização do termo de consentimento livree esclarecido. Além disso, foi ressaltado que todos os dados seriam tratados de maneira sigilosa. 0 estudo foi aprovado pelo Comitê de Ética em Pesquisa com Seres Humanos do Centro de Ciências da Saúde da Universidade Federal do Espírito Santo (UFES).

Toda a informação obtida durante a coleta de dados deu origem a um banco de dados, que foi armazenado no software M icrosoft Excel. Em seguida, foram transferidos para o programa estatístico STATA 9.0 (StataCorp), para cálculos de frequência absoluta e relativa.

\section{Resultadosediscussão}

Dos cem profissionais que concluíram o curso deespecialização em Saúdeda Família, 47 (47\%) responderam 0 instrumento da pesquisa, compondo a população deste estudo. Conforme a Tabela 1, esta população foi composta, predominantemente, do sexo feminino, com $72,3 \%$ (34), contrapondo $27,6 \%$ (13) do sexo masculino. Resultado semelhante foi observado em outros estudos, cujos autores ressaltaram o processo de feminização do trabalho em saúde nas equipes do PSF $^{5-7}$.

Em relação à idade, observou-se que a maioria dos egressos $(51,1 \%)$ encontrava-se na faixa etária de vinte a trinta anos, seguida dos que se estavam na faixa de 31 a quarenta anos $(17,8 \%)$ e de 41 a cinquenta anos (31,1\%). Gil ${ }^{5}$, ao avaliar 0 perfil dos participantes dos cursos de pós-graduação lato sensu em Saúde da Família, viabilizado pelo M inistério da Saúde com recurso do Projeto Reforço à Reorganização do SUS (REFORSUS), em 2005, encontrou resultado similar ao nosso estudo, indicando a maioria, cerca de $51,3 \%$, pertencente à faixa etária de vinte a trinta anos.

Sobre a formação profissional, conforme apresentado na Tabela 2, constatou-se que a categoria de enfermeiros foi predominante, com $40,43 \%$ (19), seguida de odontólogos e médicos, respectivamente, com $25,53 \%$ (12) e 23,4\% (11). As demais categorias (assistente social eadministração) juntas somaram 10,6\% (5). Estes resultados estão de acordo ao observado por Gil ${ }^{5}$, que constatou que a maioria dos alunos era de enfermeiros. Este autor aponta que esperava encontrar uma distribuição das categorias mais equilibrada entre enfermeiros e médicos, haja vista serem estes profissionais os queoriginalmentecompõem as equipes deSaúdeda Família. Além disso,

Tabela 1. Caracterização da população do estudo.

\begin{tabular}{lrr}
\hline \multicolumn{1}{c}{ Variáveis } & $\mathrm{N}$ & $\%$ \\
\hline Sexo & 34 & 72,3 \\
$\quad$ Feminino & 13 & 27,7 \\
M asculino & & \\
Idade $(\text { anos })^{*}$ & 23 & 51,1 \\
20 a 30 & 8 & 17,8 \\
31 a 40 & 14 & 31,1 \\
41 a 50 & & \\
"Duas respostas em branco & &
\end{tabular}

Tabela 2. Distribuição dos egressos segundo formação profissional e o tempo de formado na graduação.

\begin{tabular}{lrr}
\hline \multicolumn{1}{c}{ Distribuição } & N & \multicolumn{1}{c}{$\%$} \\
\hline Formação profissional & & \\
Enfermeiro & 19 & 40,43 \\
Odontólogo & 12 & 25,53 \\
M édico & 11 & 23,4 \\
Assistente social & 4 & 8,51 \\
Administrador & 1 & 2,13 \\
Tempo de formado* & & \\
$\quad$ de 5 anos & 24 & 53,33 \\
1 a 5 anos & 21 & 46,67 \\
\hline
\end{tabular}

${ }^{*}$ Duas respostas em branco 
afirma que a presença baixa de médicos ratifica a dificuldade encontrada para atraí-Ios para o campo deSaúde Pública. Em relação ao nosso estudo, devemos ressaltar a frequência aumentada do profissional odontólogo quando comparada ao estudo citado anteriormente, chegando a equivaler à porcentagem de médicos, mostrando, então, a inserção, embora mais recente, deste profissional na Estratégia Saúde da Família (ESF).

Quanto ao tempo de conclusão da graduação, apresentado na Tabela 2, 24 al unos estavam formados há mais de cinco anos, representando $53,33 \%$, enquanto 21 alunos, ou seja, $46,67 \%$, tinham entre um a cinco anos de formados; dois alunos não responderam. Gil ${ }^{5}$ encontrou uma porcentagem menor de recém-formados, cerca de $3,4 \%$, ao analisar somente os estudantes dos cursos de especialização.

Ao analisar as especial izações anteriores realizadas pelos profissionais, constatou-se que $56,52 \%$ (26) a possuíam, enquanto que $43,48 \%$ (20) não tinham especialização alguma. N este item, um aluno não respondeu. Ainda focalizando os estudos realizados antes da pós-graduação dePSF (PG-PSF) lacto sensu, pode-se salientar quenenhum dos profissionaishavia feito uma pós-graduação stricto sensu. A pós a realização da PG-PSF, observou-se que a maioria, ou seja, $89,36 \%$ (42) não fizeram pós-graduação do tipo lacto sensu, e apenas 10,64\% (5) a fizeram. Destes, todos a realizaram em estabelecimentos privados. Deve-se ressaltar que, posteriormente à pós-graduação de PSF, um aluno fez mestrado para Gestão Integrada em Saúde do Trabalho e M eio Ambiente.

Tabela 3. M ateriais científicos originados da PG-PSF e local de utilização.

\begin{tabular}{lrrrr}
\hline \multicolumn{1}{c}{ Produtos } & N & $\%$ & N & $\%$ \\
\hline TCC & 39 & 82,98 & & \\
Sim & 8 & 17,02 & & \\
Não & & & 36 & 94,74 \\
Local de utilização do TCC & & & 5,26 \\
$\quad$ Trabalho & & & & \\
$\quad$ Congresso e publicações & & & & \\
Outras ferramentas** & 39 & 90,7 & & \\
$\quad$ Sim & 4 & 9,3 & & \\
$\quad$ Não & & & & \\
Local de utilização das & & & 36 & 94,74 \\
outras ferramentas* & & & 2 & 5,26 \\
$\quad$ Trabalho & & & & \\
$\quad$ Trabalho e congresso & & &
\end{tabular}

*U ma resposta em branco; ** Q uatro respostas em branco.
Ao avaliarmos a utilização do trabalho de conclusão de curso (TCC) realizado na PG-PSF, pôde-se constatar que 39 profissionais (82,98\%) o utilizaram, sendo que $94,74 \%$ (36) ressaltaram que o empregaram em seu trabalho. Os demais foram utilizados em congressos e publicações, representando 5,26\% (2); apenas um aluno não respondeu (Tabela 3).

Dos profissionais analisados, 43 (91,5\%) responderam sobre a utilização de outras atividades e/ou instrumentos do curso de PG-PSF. Desses, $39(90,7 \%)$ apontaram a utilização deatividadee/ ou instrumentos do curso, enquanto quatro não as utilizaram. Para $94,74 \%$ (36), a maior parte dessas atividades foi aplicada, também, na área de trabalho, seguida de sua utilização em congressos e trabalhos, representando $5,26 \%$ (2); uma pessoa deixou de citar a sua aplicação. Estes resultados são importantes, pois permitiram observar a aplicação dos trabalhos construídos e as ferramentas adquiridas no PG-PSF nos locais de serviços de saúde, sendo esta uma das metas do PG-PSF ofertado no Estado do Espírito Santo.

É importante ressaltar que algumas estraté gias de ensino foram utilizadas duranteo curso e que nos parecem indicar uma maior apropriação e envolvimento por parte dos estudantes. Dentre estas, podemos destacar duas que foram avaliadas como de extrema importância: o uso do portfólio como técnica integrativa e autoavaliativa durante todo o processo do curso. 0 portfólio é uma coleção de todo o trabalho em andamento durante o curso relacionado com o alcance dos objetivos de cada módulo. Consisti para além dos trabalhos que foram realizados a avaliação do estudante do seu processo de ensino-aprendizagem. Este era avaliado juntamente com os coordenadores pedagógicos em momentos inter-módulos no sentido de verificar possíveis problemas de apreensão do conteúdo.

Outro ponto interessante foi que cada módulo foi precedido por uma oficina integrativa entre professores para que os conteúdos fossem alinhados e desta forma todos os cursos pudessem contar com os mesmos conteúdos de forma transversal.

Estas metodologias permitiram que houvesse um monitoramento durante todo o processo do curso.

O tipo de vínculo empregatício predominante entre os al unos antes da especialização foi o de contrato "informal", com 41,3\% (19), seguido do estatuário, com 30,43\% (14) e da CLT (Consolidação das Leis Trabalho), com 26,09\% (12). Resultado este também encontrado por $\mathrm{Gil}^{5}$, com 
predominância do contrato do tipo "informal", entendendo este como o que não assegura os direitostrabal histas consolidados na legislação. Este mesmo autor afirma quetal resultado, apesar de demonstrar o esforço de muitos gestores no processo de implementação da ESF, corrobora com as indagações a respeito da precariedade do vínculo de trabal ho na ESF, que culmina na dificuldade, elucidada pelos egressos do PG-PSF, de desenvolvimento e de continuidade dos programas e projetos voltados para 0 atendimento das necessidades explanadas pela população.

Analisan do as contribuições geradas pela PGPSF, constatou-se que $97,83 \%$ (45) dos profissionais mencionaram mudanças na suas ações de trabalho após os conhecimentos internalizados durante o curso. E, novamente, a maioria, ou seja, 95,56\% (45), afirmou que os conhecimentos adquiridos têm aplicabilidade no cotidiano do trabalho. Nos dois itens citados, apenas um aluno ficou sem responder. Estes dados fortalecem a importância dos cursos de especialização em Saúde da Família para a capacitação dos profissionais e, consequentemente, a consolidação do SUS. N este sentido, salientamos a educação permanente como o aprendizado contínuo necessário para o autoaprimoramento do sujeito na busca de competências pessoal, profissional e social, com o enfoque na potencialização pessoal rumo à aquisi ção de novos conhecimentos, conceitos eatitudes 8 .

Paim e N unes ${ }^{9}$ ressaltam que a dinamicidade e a complexificação das práticas no âmbito do trabal ho em saúde, através da incorporação constante de novas tecnologias, estabelecem padrões mutáveis nesse campo, o que requer novos mé todos de ensino e formação dos trabalhadores inseridos, com base em alguns princípios que facilitem a integração e a construção de novos saberes, sendo eles: a educação é um processo contínuo; a educação permanenteéintegral e dinâmica; a educação éum processo ordenador do conhecimento; 0 sistema integrativo deve ter caráter integrador; a educação é inovadora e o conhecimento se origina nas necessidades ou problemas sociais dentro de um projeto histórico. Assim, os cursos e programas de capacitação e aperfeiçoamento em Saúde Coletiva devem estar voltados para as mudanças e tendências no âmbito das práticas que são produzidas no cotidiano mutável dos serviços de saúde.

No que tange ao aumento salarial, 45 dos entrevistados, cerca de $95,74 \%$, ressaltaram que não o obtiveram. I sso aconteceu para apenas 4,56\% (2) da população pesquisada. Em relação à melhora nas condições de trabalho, dos 44 respondentes, $75 \%$ (33) não a tiveram concretizada; já 25\% (11) constataram essa melhoria.

Acerca das contribuições proporcionadas pelo curso, 84,78\% (39) asseguraram melhora na capacidade de empregabilidade, com apenas um aluno sem responder o item. Todos os 47 respondentes declararam que as ferramentas oferecidas pelo curso proporcionaram qualidade no desempenho de suas funções.

Procurando-se investigar as competências adquiridas pelos entrevistados no decorrer do curso, 95,65\% (44) informaram ter adquirido reflexão sobre as influências na saúde do indivíduo eda coletividade perante a família; um aluno deixou de responder. Cerca de 77,78\% (35) dos entrevistados afirmaram, também, conseguir atuar junto à família na implementação de ações de saúde nos níveis primários, secundários e terciários, com baseno atendimento prioritário das reais necessidades de saúde expressas pelos indivíduos no cerne da comunidade.

A proposta de um atendimento integral focado no espaço da família surge com a proposição do Programa de Saúde Família, atualmente denominado como Estratégia de Saúde da Família (ESF), implantado no país a partir de 1994, que reafirma os princípios do SUS e, para isso, fundamenta-se na concepção de atenção focalizada na promoção da qualidade de vida, tendo como objetivo trabalhar práticas de saúde que gerem integração entre as ações individuais e coletivas. Os pressupostos que devem nortear a organização das práticas de saúde voltadas à família, tais como o reconhecimento da saúde como direito de cidadania, a el eição da família e seu espaço social como núcleo básico de abordagem, a prestação de atenção integral, a humanização das práticas de saúde e a partici pação comunitária, são parte de uma estratégia primordial para induzir mudanças, tanto pela interferência sobre a dinâmica do mercado de trabalho em saúde quanto pela reestruturação do processo de trabalho ${ }^{10}$.

A estratégia nasce com o propósito de superar o modelo de assistência vigente, responsável pela ineficiência do setor, insatisfação dos usuários, desqualificação profissional einiquidades ${ }^{11}$. Assim, seu objetivo seria reorganizar as práticas assistenciais em novas bases e critérios, com a compreensão ampla do processo saúde doença e da necessidade de intervenções que ultrapassem as práticas curativas, com uma atuação profissional dinâmica fundamentada na análise ena avaliação das ações ena elaboração de planos de 
intervenção fundamentados no diagnóstico situacional deum território definido a priori ecom base no enfoque de riscos.

A maioria da população em estudo, representando $91,40 \%$ (43), ressaltou que consegue planejar suas ações baseadas no perfil epidemiológico da área em que trabalha, e 85,10\% (40) planejam, também, suas ações com ênfase no enfoque de risco, depois de ter passado pelo curso.

Dos 44 profissionais que responderam o item relacionado ao gerenciamento de recursos humanos, 70,45\% (31) asseguram ter essa competência na área de abrangência da equipe. Trinta profissionais $(66,67 \%)$ ressaltaram a aquisição da capacidade de gerenciar os recursosmateriais; doisnão responderam. E a maioria dos profissionais, re presentando 71,74\% (33), não sesentecapacitada no gerenciamento de recursos financeiros, com apenas uma resposta não emitida.

Dos 44 respondentes, 27 (61,36\%) informaram ter adquirido o estímulo para desenvolver pesquisas nas equipes de Saúde da Família, enquanto dezessete (38,64\%) não o confirmaram. E 91,30\% (42) asseguram ter competência para realizar diagnóstico situacional de saúde da população; um profissional não respondeu.

$80,43 \%$ (37) dos 46 respondentes declararam ter adquirido competência em relação à atuação nos serviços de saúde, implantando e/ou implementando ações nos três níveis de atenção. Verificou-se, também, que 93,18\% (41) obtiveram a competência para o estímulo ea implementação da participação da comunidade nas atividades de promoção da Saúde da Família. Vale lembrar que, dos 47 entrevistados, apenas três ficaram sem responder a essa questão.

No que se refere à dinamização e à imple mentação de sistema de referência entre as Unidades de Saúde (US), observou-se que $66,67 \%$ (30) dos entrevistados adquiriram tal competência, enquanto que $33,33 \%$ (15) não a alcançaram. Fato semel hante ocorreu ao se tratar de sistema de contrarreferência: $57,78 \%$ (26) conseguiram competência para tal, enquanto $42,22 \%$ (19) não. Dois entrevistados deixaram de responder a ambas as questões. Ao setratar do aprimoramento da capacidade de trabalho em equipe de forma interdisciplinar, constatou-se que a maioria dos entrevistados, $97,67 \%$ (42), sente-se capacitada. Nessa questão, ficou em branco a resposta de quatro entrevistados.

Em relação ao assumir o papel de educador, $44(93,61 \%)$ dos participantes indicaram que se sentem preparados para assumir tal cargo e compartilhar o seu saber. Quarenta e três responde- ram à questão que adota a metodologia de trabalho para tomar decisões no seu processo profissional e 93,02\% (40) afirmaram adotar tal medida na execução do seu ofício.

\section{Consideraçõesfinais}

Os resultados desta pesquisa revelaram o perfil dos trabalhadores de saúde do Estado do Espírito Santo egressos do curso de PG-ESF, sendo predominantemente do sexo feminino, de adultos jovens, na faixa etária de vinte a trinta anos. Além disso, a maioria era de enfermeiros e com mais de cinco anos de formados.

Quanto às contribuições geradas pela realização do curso, observaram-se muitos relatos referentes às mudanças nas ações de trabalho após os conhecimentos internalizados durante o PGESF. A maioria afirmou que os conhecimentos adquiridos tiveram aplicabilidadeno cotidiano do trabal ho e grande parte dos entrevistados conseguiu, após o curso, planejar suas ações com base no perfil epidemiológico da área onde atua.

Os resultados nos permitiram constatar a relevância do curso para a capacitação profissional dos alunos, com a perspectiva de constante adequação dos conteúdos e aperfeiçoamento do processo didático-pedagógico, através de um sistema de educação continuada ou até de educação permanente, bem como de processos de acompanhamento e supervisão em serviço.

No entanto, é preciso apontar as limitações do estudo principalmente em relação ao instrumento utilizado. Como o instrumento apenas verificou as ações do ponto de vista dos estudantes, este pode estar sujeito ao viés de informação, uma vez que os mesmo questionamentos feitos a outros atores (por exemplo, aos gerentes das unidades em que trabal ham os profissionais especializandos, indagando sobre mudanças ou inovações nas práticas de saúde e aos seus colegas de equipe) poderiam fornecer informaçõesquetivessem maior capacidade de revelar aspectos de mudanças de práticas de saúde. Face ao exposto, recomendamos que estudos que pretendam avaliar egressos incluam no seu escopo outros profissionais envolvidos na rede profissional dos egressos de cursos tanto lato sensu quanto scrito sensu.

Deve-se ressaltar ainda que os problemas inerentes à fragilidade dos vínculos empregatícios, atrelados à baixa remuneração dos trabalhadores de saúde em questão, põem em risco os investimentos empregados na concretização do curso, pois os programas e mudanças realizados 
pelos profissionais de saúde no local de trabal ho deixam de ter continuidade ou não são implementados devido à insatisfação e à grande rotatividade profissional, com a consequentedificuldadena construção de vínculos entre os profissionais e a população adscrita. Por isso, uma política de educação permanente, mesmo que privilegi eformas de qualificação ecapacitação profis- sional, tal como cursos de pós-graduação lato sensu, deve também estar atenta e propor meios de incentivo a formas de contratação mais sólidas e comprometidas com a valorização do trabalhador, fornecendo instrumentos e estímulos para que os municípios criem e apliquem estratégias de desenvolvimento de pessoas e gestão coadunadas com os princípios e objetivos do PSF.

\section{Colaboradores}

ELN M aciel eRCD Lima participaram da elaboração do tema, construção do quadro teórico, coleta dos dados, análise e delineamento final do estudo. PF Figueiredo participou da coleta dos dados, construção do quadro teórico e revisão final. TN Prado participou da coleta dos dados, construção do quadro teórico, análise e revisão final. HS Galavote participou da coleta dos dados e revisão final. MC Ramose M D Araújo participaram da elaboração do tema e revisão final.

\section{Referências}

1. Hartz ZMA, organizadora. Avaliação em Saúde: dos modelos conceituais à prática na análise da implantação dos programas. Rio de Janeiro: ENSP/Fiocruz; 1997.

2. Akerman M, Luiz OC. Avaliação da atenção primária: uma breve aproximação de conceitos e caminhos. In: Brasil. CONASS. Acompanhamento e avaliação da atenção primária. Vol. 1. 1ạ ed. Brasília: CONASS; 2004. p. 42-74.

3. Cotta TC. M etodologia de avaliação de programas e projetos sociais: análise de resultados e impactos. Rev Serv Pub 1998; 49(2):32-38.

4. Finger AP, Moreira EC. Evaluación académica en instituciones universitarias de América Latina: análisis de algunas experiencias. Córdoba: Dirección General de Publicaciones dela Universidad Nacional de Córdoba; 1989. p.88.

5. Gil CRR. Formação de recursos humanos em saúde da família: paradoxos e perspectivas. Cad Saude Publica 2005; 21(2):490-498.

6. Escorel S. Avaliação da implementação do Programa de saúde da Família em dez grandes centros urbanos [relatório final]. Brasília: M inistério da Saúde; 2002.

7. Girardi SN, Carvalho CL. Mercado de Trabalho e regulação das Profissões de Saúde. In: Negri B, Faria $R$, Viana ALD, organizadores. Recursos humanos em saúde: política, desenvolvimento e mercado de trabalho. Campinas: Editora Unicamp; 2002. p. 221-256.

8. Paschoal AS, Mantovani MF, Méier MJ. Percepção da educação permanente, continuada e em serviço para enfermeiros de um hospital de ensino. Rev. Esc. Enferm. USP [periódico na Internet] 2007 [acessado 2008 jan 29];41(3):[cerca de 7 p.]. Disponível em: http://www.scielo.br/pdf/reeusp/v41n3/19.pdf 
9. Paim JS, Nunes TCM . Contribuições para um programa de educação continuada em saúde coletiva. Cad Saude Publica 1992; 8(3):262-269.

10. Brito JC. O trabalho em saúde: olhando e experienciando o SUS no cotidiano. Cad Saude Publica 2005; 21(5):1612-1614.

11. Franco TB, Bueno WS, M erhy EE. O acolhimento e os processos de trabalho em saúde: 0 caso de Betim (MG). In: Merhy EE, Magalhães Jr. H M, Rimoli J, Franco TB, Bueno WS. 0 trabalho em saúde: olhando e experenciando o SUS no cotidiano. São Paulo: Hucitec; 2003. p. 37-54.

Artigo apresentado em 27/04/2008

Aprovado em 31/10/2008

Versão final apresentada em 04/11/2008 